\title{
EXISTENCE AND REGULARITY OF SOLUTIONS TO ELLIPTIC CALCULUS OF VARIATIONS PROBLEMS AMONG SURFACES OF VARYING TOPOLOGICAL TYPE AND SINGULARITY STRUCTURE
}

\author{
BY F. J. ALMGREN, JR. ${ }^{1}$
}

Communicated by Herbert Federer, March 6, 1967

Definitions and Notation. (1) $m$ and $n$ denote positive integers.

(2) $H^{k}$ denotes Hausdorff $k$ dimensional measure in $R^{m+n}$ for $k=m, m-1$.

(3) $G_{m}^{m+n}$ denotes the Grassmann manifold of unoriented $m$ plane directions in $R^{m+n}$ (which can be regarded as the space of all unoriented $m$ planes through the origin in $R^{m+n}$ ).

(4) A $C^{(k)}$ integrand [real analytic integrand] is a function [real analytic function] $F: G_{m}^{m+n} \rightarrow R \cap\{t: t>0\}$ whose partial derivatives up to order $k$ exist and are continuous. Here $k$ denotes either a positive integer or $\infty .^{2}$

(5) A surface $S$ is a compact $m$-rectifiable subset of $R^{m+n}$. If $S$ is a surface, then, for $H^{m}$ almost all $x \in S, S$ has an approximate tangent $m$ plane direction at $x$, denoted $\mathrm{S}(x)$.

(6) The integral of an integrand $F$ over a surface $S$ is defined to be

$$
F(S)=\int_{x \in S} F(\mathrm{~S}(x)) d H^{m} x .
$$

(7) A boundary $B$ is a compact $(m-1)$-rectifiable subset of $R^{m+n}$ with $\boldsymbol{H}^{m-1}(B)<\infty$.

(8) $G$ denotes the category of all finitely generated abelian groups. If $B$ is a boundary, $S$ is a surface, and $G \in G$, we denote by $H_{m-1}(B ; G)$ and $H_{m-1}(B \cup S ; G)$ the $m-1$ dimensional Vietoris homology groups of $B$ and $B \cup S$, respectively, with coefficients in $G$. If $\sigma \in H_{m-1}(B ; G)$ we say that $S$ spans $\sigma$ if and only if $i_{*}(\sigma)=0$ where

$$
i_{*}: H_{m-1}(B ; G) \rightarrow H_{m-1}(B \cup S ; G)
$$

is induced by the inclusion $i: B \rightarrow B \cup S$.

\footnotetext{
1 This research was supported in part by grant NSF-GP 2425 from the National Science Foundation.

${ }^{2}$ The existence and regularity results of this paper have recently been extended to apply to integrands $F: R^{m+n} \times G_{m}{ }^{m+n} \rightarrow R$ which are elliptic on each tangent space. For such integrands one sets $F(S)=\int F(x . S(x)) d\left(H^{m} S\right) x$.
} 
Elliptic integrands. Suppose $G \in \mathbf{G}$ and $D$ is a closed $m$ disk in $R^{m+n}$. Let $\partial D$ denote the boundary $m-1$ sphere of $D$ and let $\sigma$ $\in H_{m-1}(\partial D ; G)-\{0\}$. As an hypothesis on our integrand $F$ we need to require that the unique surface which spans $\sigma$ and minimizes $F$ be $D$ itself, and that this be true in a uniform way. More precisely, we call the integrand $F$ elliptic with respect to $G$ if and only if there exists a positive number $c$ such that if $D$ is any closed $m$ disk in $R^{m+n}$, $\sigma \in H_{m-1}(\partial D ; G)-\{0\}$, and $S$ is a surface which spans $\sigma$, then

$$
F(S)-F(D) \geqq c\left[H^{m}(S)-H^{m}(D)\right] .
$$

The ellipticity of $F$ with respect to any $G \in G$ is equivalent to the uniform convexity of $F$ if $n=1$, and $\left(^{*}\right)$ is implied by the uniform convexity of $F$ for arbitrary $n$ when $S$ is orientable and has $\partial D$ as its oriented boundary. The class of integrands $F$ which are elliptic with respect to all $G \in G$ forms a convex set in the space of all functions $G_{m}^{m+n} \rightarrow R$ which set, in particular, contains a neighborhood of the $m$ area integrand (i.e. $F \equiv 1$ ) in the $C^{(2)}$ topology. Also if $L: R^{m+n} \rightarrow R^{m+n}$ is a nonsingular linear mapping, then $L_{\sharp} F$ is elliptic with respect to $G \in G$ if and only if $F$ is.

Regularity of surfaces. Let $S$ be a surface and $k$ be either a positive in teger or $\infty$. We say that $S$ is $C^{(k)}$ regular almost everywhere [real analytic almost everywhere] if and only if there is a compact subset $T$ of $S$ with $H^{m}(T)=0$ such that $S \sim T$ is a $k$ times continuously differentiable [real analytic] $m$ dimensional submanifold of $R^{m+n}$.

Examples show that, except possibly for $n=1$ and $G$ having the integers modulo two as a direct summand, a surface $S$ minimizing an elliptic integrand may have singularities of dimension $m-1$. Present techniques are not adequate to show that the singular set $T \sim B$ (for a suitable boundary $B$ ) is regular or, even locally, of finite $\boldsymbol{H}^{m-1}$ measure.

THEOREM 1. Let $k \geqq 4$ be either a positive integer or $\infty$ and $G \in G$. Suppose $F$ is a $C^{(k)}$ integrand [real analytic integrand] which is elliptic with respect to $G, B$ is a boundary, and $\sigma \in H_{m-1}(B ; G)$. Then there exists a surface $S$ with the following properties:

(1) $S$ spans $\sigma$.

(2) $F(S) \leqq F(T)$ whenever $T$ is a surface which spans $\sigma$.

(3) $S$ is $C^{(k-1)}$ regular almost everywhere [real analytic almost everywhere].

Remark. For some applications one wishes to know that, for a prescribed boundary $B$, there exists among all surfaces having $B$ as 
boundary in a topologically non trivial way (either locally or globally) a surface minimizing the integral of an integrand. Figure 1.6 in [A2] shows an example due to J. F. Adams of a soap film which, although locally minimizing area, nevertheless retracts onto its boundary. In the following sections we indicate some compactness and regularity properties of surfaces which minimize the integral of an elliptic integrand in a topological sense weaker than that required in Theorem 1.

Definitions. (1) Suppose $S$ is a surface and $B$ is a boundary. We call $T$ a comparison surface for $S$ with respect to $B$ if and only if $T$ is the image of $S$ under a Lipschitzian deformation which keeps $B$ fixed. Clearly if $S$ spans $\sigma \in H_{m-1}(B ; G)$ for some $G \in G$ and $T$ is a comparison surface for $S$ with respect to $B$, then $T$ also spans $\sigma$.

(2) Suppose $S$ is a surface, $B$ is a boundary, $F$ is an integrand, and $U=\left\{U_{1}, U_{2}, U_{3}, \cdots\right\}$ is an open covering of $R^{m+n}$. We say that $S$ is $F$ minimal with respect to $B$ and $U$ if and only if $F(S) \leqq F(T)$ whenever $T$ is a comparison surface for $S$ and there is a positive integer $j$ such that $S \sim U_{j}=T \sim U_{j}$. We say that $S$ is $F$ minimal with respect to $B$ if and only if $S$ is $F$ minimal with respect to $B$ and $\left\{R^{m+n}\right\}$.

(3) We call an integrand $F$ elliptic (without reference to a coefficient group) if and only if there exists a positive number $c$ such that

$$
F(S)-F(D) \geqq c\left[H^{m}(S)-H^{m}(D)\right]
$$

whenever $D$ is an $m$ disk in $R^{m+n}$ and $S$ is a surface which cannot be deformed into $\partial D$ by a Lipschitzian map leaving $\partial D$ fixed. As before, the ellipticity of $F$ is equivalent to the uniform convexity of $F$ if $n=1$, and the class of elliptic integrands forms a convex set in the space of all functions $G_{m}^{m+n} \rightarrow R$ which set, in particular, contains a neighborhood of the $m$ area integrand in the $C^{(2)}$ topology. Also if $L: R^{m+n} \rightarrow R^{m+n}$ is a nonsingular linear mapping, then $L_{\sharp F}$ is elliptic if and only if $F$ is.

(4) Each surface $S$ is naturally a measure $|S|$ on $R^{m+n} \times G_{m}^{m+n}$ (called the integral varifold associated with $S$ ) given by $|S|$ $=\phi_{\#}\left(H^{m} \cap S\right)$ where $\phi: S \rightarrow R^{m+n} \times G_{m}^{m+n}$ sends $H^{m}$ almost all $x \in S$ to $(x, \mathrm{~S}(x))$. The weak topology on the space of all Radon measures on $R^{m+n} \times G_{m}^{m+n}$ induces the weak topology on the space of all surfaces.

TheOREM 2. Let $k \geqq 4$ be either a positive integer or $\infty$. Suppose $F$ is $a C^{(k)}$ integrand [real analytic integrand] which is elliptic, $B$ is a boundary, $U$ is an open covering of $R^{m+n}$, and $0<\mu<M<\infty$.

(1) If $S$ is a surface which is $F$ minimal with respect to $B$ and $U$, then $S$ is $C^{(k-1)}$ regular almost everywhere [real analytic almost everywhere]. 
(2) The space of all surfaces $S$ which are $F$ minimal with respect to $B$ and $U$ and for which $F(S) \leqq M$ is compact in the weak topology and $F$ is a continuous function on this space.

(3) As a consequence of (1) and (2) we see that if $F(T) \geqq \mu$ whenever $T$ is an $F$ minimal surface with respect to $B$ and $U$ and $F(T)>0$, then there exists a surface $S$ which is $F$ minimal with respect to $B$ and $U$ and hence $C^{(k-1)}$ regular almost everywhere [real analytic almost everywhere] such that $F(S) \leqq F(T)$ whenever $F(T)>0$ and $T$ is an $F$ minimal surface with respect to $B$ and $U$.

REMARK. Conditions sufficient to imply a positive lower bound to the positive numbers $F(T)$ for surfaces $T$ which are $F$ minimal with respect to $B$ and $U$ of ten arise in individual problems. One sufficient condition is that $B$ be a Lipschitz neighborhood retract (which would be true if $B$ were a compact differentiable $m-1$ dimensional submanifold of $R^{m+n}$ ) and $U=\left\{R^{m+n}\right\}$. See $[\mathrm{A} 1,11.1(4,5), 11.3,11.4,11.5]$ [A2, Figures 1-6, 1-9, 4-7].

REMARK. In case $G$ is the group of integers, $n=1$, and $F \equiv 1$, the regularity almost everywhere of $S$ minimizing $F$ can be inferred from the work of E. De Giorgi [D] (see also M. Miranda [M]). In case $G$ is a finite abelian group and $F \equiv 1$, the existence and regularity almost everywhere of $S$ spanning $\sigma$ and minimizing $F$ as in Theorem 1 was proved by the late E. R. Reifenberg [R1, R2, R3] and extended to manifolds by C. B. Morrey [MO]. It does not seem possible to generalize the arguments of De Giorgi or of Reifenberg to general elliptic integrands. In particular, the orthogonal invariance of the $m$ area integrand $F \equiv 1$ is essential for the applicability of Reifenberg's methods.

The present results are geometrically and measure theoretically based on the integral currents of H. Federer and W. H. Fleming [FF], the flat chains of Fleming [F], and integral varifolds [A1]. Basic analytic estimates for first derivatives of $F$ minimal surfaces come from bounds on the first and second derivatives of distribution solutions to systems of second order linear elliptic partial differential equations with constant coefficients, for help with which I am indebted to L. Hörmander. Higher differentiability of the surfaces follows from the work of C. B. Morrey [MO].

\section{REFERENCES}

A1. F. J. Almgren Jr., The theory of varifolds. A variational calculus in the large for the $k$ dimensional area integrand, (mimeographed), 1965.

A2. - Plateau's problem. An invitation to varifold geometry, W. A. Benjamin, Inc., New York, 1966. 
D. E. De Giorgi, Frontiere orientate di misura minima, Sem. di Mat. de Scuola Norm. Sup. Pisa, 1960-1961, 1-56.

FF. H. Federer, and W. H. Fleming, Normal and integral currents, Ann. of Math. 72 (1960), 458-520.

F. W. H. Fleming, Flat chains over a finite coefficient group, Trans. Amer. Math. Soc. 121 (1966), 160-186.

M. M. Miranda, Sul minimo dell' integrale del gradiente di una funzione, Ann. Scuola Norm. Sup. Pisa (3) 19 (1965), 626-665.

MO. C. B. Morrey, Multiple integrals in the calculus of variations, Springer-Verlag, New York, 1966.

R1. E. R. Reifenberg, Solution of the Plateau problem for m-dimensional surfaces of varying topological type, Acta Math. 104 (1960), 1-92.

R2. - An epiperimetric inequality related to the analyticity of minimal surfaces, Ann. of Math. 80 (1964), 1-14.

R3. - On the analyticity of minimal surfaces, Ann. of Math. 80 (1964), 1521.

\section{Princeton University}

\section{ZERO-SETS IN POLYDISCS ${ }^{1}$}

\section{BY WALTER RUDIN}

Communicated by Maurice Heins, Feb. 10, 1967

For $N=1,2,3, \cdots$ the polydisc $U^{N}$ consists of all $z=\left(z_{1}, \cdots, z_{N}\right)$ in the space $C^{N}$ of $N$ complex variables whose coordinates satisfy $\left|z_{j}\right|<1$ for $j=1, \cdots, N$. We write $U$ for $U^{1}$. The distinguished boundary of $U^{N}$ is the torus $T^{N}$ defined by $\left|z_{j}\right|=1(1 \leqq j \leqq N)$. The zero-set of a complex function $f$ defined in $U^{N}$ is the set $Z(f)$ of all $z \in U^{N}$ at which $f(z)=0$. We call a set $E \subset U^{N}$ a zero-set in $U^{N}$ if $E=Z(f)$ for some $f$ which is holomorphic in $U^{N}$. The main result of this note gives a sufficient condition for zero-sets of bounded functions.

THEOREM 1. If $E$ is a zero-set in $U^{N}$ and if no point of $T^{N}$ is a limit point of $E$, then there is a bounded holomorphic function $F$ in $U^{N}$ such that $Z(F)=E$.

[The term "limit point" refers of course to the topology induced on $C^{N}$ by the euclidean metric.]

For $N=1$ this is utterly trivial since the hypothesis then forces

${ }^{1}$ Research sponsored by the Air Force Office of Scientific Research, Office of Aerospace Research, United States Air Force, under AFOSR Grant No. 1160-66, and by the Wisconsin Alumni Research Foundation. 